\title{
Some Remarks on the Notion of Contraction of Lie Group Representations
}

\author{
Benjamin CAHEN
}

\begin{abstract}
In the series of papers [1-4], L. Barker developed a general notion of convergence for sequences of Hilbert spaces and related objects (vectors, operators...). In this paper, we remark that Barker's convergence for sequences of operators provides a notion of contraction of Lie group (unitary) representations and we compare it to the usual one introduced by J. Mickelsson and J. Niederle. This allows us to illustrate Barker's convergence of operators by various examples taken from contraction theory.
\end{abstract}

\section{INTRODUCTION}

In the pioneering paper [19], Inönü and Wigner introduced the notion of contraction of Lie groups and Lie group representations on physical grounds: If two physical theories are related in a limiting process, then the associated invariance groups and their representations should be also related in a limiting process called contraction. For instance, the Galilei group is a contraction of the Poincaré group [19].

Contractions of Lie algebras, Lie groups and their representations have been studied by many authors and continue to be a subject of active research, see for instance the papers [17], [18] and their references.

In fact, the systematic study of contractions of Lie group representations began with the work of Mickelsson and Niederle. In [22], a proper definition of the contraction of unitary representations of Lie groups was given for the first time and was illustrated by various examples, including contractions of the principal series representations of $S O_{0}(n+1,1)$ to the non-zero mass representations of the Euclidean group $\mathbb{R}^{n+1} \rtimes S O(n+1)$ and to the positive mass-squared representations of the Poincaré group $\mathbb{R}^{n+1} \rtimes S O_{0}(n, 1)$. More generally, in [16], Dooley and Rice established a contraction of the principal series representations of a semi-simple Lie group to some unitary irreducible representations of its Cartan motion group.

2000 Mathematics Subject Classification. 22E45; 22E46; 81R05.

Key words and phrases. Contractions; Lie groups; unitary representations; sequences of Hilbert spaces. 
In [14] and [13], contractions of representations were interpreted in the setting of the Kirillov-Kostant method of orbits [20] and, in [13] and [5]-[10], Berezin and Weyl quantization maps were used in order to obtain contraction results.

Recently, in the series of papers [1]-[4], L. Barker developed a general theory of convergence for a sequence $\left(\mathcal{H}_{n}\right)$ of Hilbert spaces in order to describe continuum quantum systems as limits of discrete quantum systems. This theory includes a notion of convergence for a sequence $f_{n} \in \mathcal{H}_{n}$ and for a sequence $\left(A_{n}\right)$ where $A_{n}$ is a bounded operator on $\mathcal{H}_{n}$ for each integer $n>0$.

Although this is not said explicitly in [3], it appears that Barker's convergence of operators immediately gives a new notion of contraction of Lie group unitary representations. The main goal of the present paper is then to compare this notion to the usual one introduced in [22].

This paper is organized as follows. In Section 2, we recall some basic facts about contractions and, in Section 3, we outline Barker's theory. In Section 4 , we establish our main results. We compare the notion of contraction of Lie group unitary representations which derives from Barker's theory with that of Mickelsson and Niederle. In particular, we show that a contraction of Lie group representations in the sense of [22] is also a contraction in the sense of Barker's theory, the converse being true under some additional assumptions. In Section 5, we give some examples of contractions and we mention some open questions.

\section{Generalities on Contractions}

In this section, we review some basic facts on contractions. The material of this section is essentially taken from [22] (see also [8]). We begin by recalling the definitions of a Lie algebra contraction and a Lie group contraction.

Let $G$ and $H$ be two real Lie groups with Lie algebras $\mathfrak{g}$ and $\mathfrak{h}$, respectively. We assume that $\mathfrak{g}$ and $\mathfrak{h}$ have the same dimension and we denote by $[\cdot, \cdot]_{1}$ and $[\cdot, \cdot]_{0}$ the Lie brackets on $\mathfrak{g}$ and $\mathfrak{h}$, respectively.

Definition 2.1. A contraction of $\mathfrak{g}$ to $\mathfrak{h}$ is a family $\left(C_{r}\right)_{r \in] 0,1]}$ of linear isomorphisms from $\mathfrak{g}$ onto $\mathfrak{h}$ such that

$$
\lim _{r \rightarrow 0} C_{r}^{-1}\left[C_{r}(X), C_{r}(Y)\right]_{1}=[X, Y]_{0}
$$

for all $X$ and $Y$ in $\mathfrak{h}$.

Definition 2.2. A contraction of $G$ to $H$ is a family $\left(c_{r}: V \rightarrow G\right)_{r \in] 0,1]}$ of smooth maps defined on the same neighborhood $V$ of the identity element $e_{H}$ of $H$ satisfying the properties:

(1) For each $r \in] 0,1], c_{r}$ maps $e_{H}$ to the identity element $e_{G}$ of $G$;

(2) There exists an open neighborhood $W$ of $e_{G}$ such that $c_{r}$ is a diffeomorphism of $c_{r}^{-1}\left(W^{2}\right)$ onto $W^{2}$ for each $\left.\left.r \in\right] 0,1\right]$; 
(3) For each $x \in V$ there is $\left.\left.r_{x} \in\right] 0,1\right]$ such that $c_{r}(x) \in W$ for $r<r_{x}$;

(4) For all $x, y$ in $V$, we have

$$
\lim _{r \rightarrow 0} c_{r}^{-1}\left(c_{r}(x) \cdot c_{r}(y)\right)=x y .
$$

Because of (2) and (3), the expression that is taken to the limit in (4) is well defined for $r<r_{x}, r_{y}$. If the family $\left(c_{r}\right)_{r \in] 0,1]}$ is a contraction of $G$ to $H$ then the family $\left(d c_{r}\left(e_{H}\right)\right)_{r \in] 0,1]}$ is a contraction of $\mathfrak{g}$ to $\mathfrak{h}$. Conversely, if $\left(C_{r}\right)_{r \in] 0,1]}$ is a contraction of $\mathfrak{g}$ to $\mathfrak{h}$ such that the family $\left.\left.\left(\left\|C_{r}\right\|_{\text {op }}, r \in\right] 0,1\right]\right)$ is bounded then by adapting arguments of the proof of Theorem 2.15.4 in [24] one can show that the family $\left(C_{r}\right)_{r \in] 0,1]}$ exponentiates to a contraction $\left(c_{r}=\exp _{G} \circ C_{r} \circ \exp _{H}^{-1}\right)_{r \in] 0,1]}$ of $G$ to $H$.

Now, we fix a contraction $\left(c_{r}\right)_{r \in] 0,1]}$ of $G$ to $H$ as in Definition 2.2. For each integer $n>0$ let $\pi_{n}$ be a unitary representation of $G$ on a Hilbert space $\mathcal{H}_{n}$. Let $\rho$ be a unitary representation of $H$ in a Hilbert space $\mathcal{H}$. We denote by $\langle\cdot, \cdot\rangle_{n}$ and $\langle\cdot, \cdot\rangle$ the scalar products on $\mathcal{H}_{n}$ and $\mathcal{H}$ and by $\|\cdot\|_{n}$ and $\|\cdot\|$ the corresponding norms.

Definition 2.3. We say that the representation $\rho$ is a MN-contraction of the sequence $\left(\pi_{n}\right)$ if there exists a sequence $\left.\left.r(n) \in\right] 0,1\right]$ with limit 0 , a sequence of unitary operators $A_{n}: \mathcal{H}_{n} \rightarrow \mathcal{H}$ and a dense subspace $D$ of $\mathcal{H}$ satisfying the following properties:

(1) For each $f \in D$ there exists an integer $n_{0}>0$ such that for each $n \geq n_{0}$ we have $f \in A_{n}\left(\mathcal{H}_{n}\right)$;

(2) For each $f \in D$ and $h \in V$, we have

$$
\lim _{n \rightarrow+\infty}\left\|A_{n} \pi_{n}\left(c_{r(n)}(h)\right) A_{n}^{-1} f-\rho(h) f\right\|=0 .
$$

Note that the expression that is taken to the limit in Definition 2.3 is well-defined for $n \geq n_{0}$.

Remark 2.4. Let $f_{1}$ and $f_{2}$ in $D$. Clearly, since the operators $A_{n}$ are unitary, Property (2) of Definition 2.3 implies that

$$
\lim _{n \rightarrow+\infty}\left\langle\pi_{n}\left(c_{r(n)}(h)\right) A_{n}^{-1} f_{1}, A_{n}^{-1} f_{2}\right\rangle_{n}=\left\langle\rho(h) f_{1}, f_{2}\right\rangle
$$

for each $h \in V$. Conversely, assume that (2.1) holds for each $f_{1}, f_{2} \in$ $D$ and each $h \in V$. Let $f \in D$ and $h \in V$. Then we immediately see that $\left(A_{n} \pi_{n}\left(c_{r(n)}(h)\right) A_{n}^{-1} f\right)$ converges weakly to $\rho(h) f$ in $\mathcal{H}$. Since $\left\|A_{n} \pi_{n}\left(c_{r(n)}(h)\right) A_{n}^{-1} f\right\|=1=\|\rho(h) f\|$, we have that $\left(A_{n} \pi_{n}\left(c_{r(n)}(h)\right)\right.$ $A_{n}^{-1} f$ ) converges strongly to $\rho(h) f$ in $\mathcal{H}$.

Remark 2.5. Here we mention two important particular cases of MNcontractions.

1) The case when $A_{n}\left(\mathcal{H}_{n}\right)=\mathcal{H}$ for each $n$. In that case, we can assume that $D=\mathcal{H}$ in Definition 2.3. Indeed, if Property (2) of Definition 2.3 holds for each $f \in D$ then Property (2) also holds for each 
$f \in \mathcal{H}$. Moreover, if $\left(f_{p}\right)$ is an orthonormal basis of $\mathcal{H}$, then for each $n, f_{p}^{n}=A_{n}^{-1} f_{p}$ is an orthonormal basis for $\mathcal{H}_{n}$ and, according to Remark 2.4, we see that Property (2) of Definition 2.3 is equivalent to the fact that

$$
\lim _{n \rightarrow+\infty}\left\langle\pi_{n}\left(c_{r(n)}(h)\right) f_{p}^{n}, f_{q}^{n}\right\rangle_{n}=\left\langle\rho(h) f_{p}, f_{q}\right\rangle
$$

for each $h \in V$ and each $p, q$. An example of such a situation is given in Section 5.

2) The case when $\mathcal{H}_{n}$ is finite-dimensional for each $n$. See in particular [15], [23], [6] and [9].

Remark 2.6. In the setting of Definition 2.3, note that, since the operators $A_{n}$ are unitary, $A_{n}\left(\mathcal{H}_{n}\right)$ is a closed subspace of $\mathcal{H}$ for each $n$. Let $R_{n}$ be the operator from $\mathcal{H}$ to $\mathcal{H}_{n}$ defined by $R_{n} f=A_{n}^{-1} f$ if $f \in A_{n}\left(\mathcal{H}_{n}\right)$ and $R_{n} f=0$ if $f$ is orthogonal to $A_{n}\left(\mathcal{H}_{n}\right)$. Then we have

$$
\lim _{n \rightarrow+\infty}\left\langle R_{n} f_{1}, R_{n} f_{2}\right\rangle_{n}=\left\langle f_{1}, f_{2}\right\rangle
$$

for each $f_{1}, f_{2} \in D$.

In the literature, we can also find the following notion of contraction of Lie group unitary representations which is weaker than MN-contraction (see for instance [12]).

Definition 2.7. If, in Definition 2.3, we replace the condition that the operators $A_{n}$ are unitary by the condition that the operators $A_{n}$ are injective, continuous, satisfying $\lim _{n \rightarrow+\infty}\left\|A_{n}^{-1} f\right\|_{n}=\|f\|$ for each $f \in D$, then we say that $\rho$ is a nuMN-contraction of $\left(\pi_{n}\right)$.

\section{BARKER'S THEORY}

In this section, we outline Barker's theory. See [1], [3] and, for applications to Physics, [2] and [4].

As in Section 2, we consider a sequence $\left(\mathcal{H}_{n}\right)$ of Hilbert spaces, a Hilbert space $\mathcal{H}$ and a dense subspace $D$ of $\mathcal{H}$. Let $R_{n}: D \rightarrow \mathcal{H}_{n}$ be a sequence of linear maps satisfying

$$
\langle f, g\rangle=\lim _{n \rightarrow+\infty}\left\langle R_{n} f, R_{n} g\right\rangle_{n}
$$

for each $f, g \in D$. The family $\left(\mathcal{H}_{n}, R_{n}\right)$ is then called an inductive resolution of $\mathcal{H}$.

Definition 3.1 ([1]). Let $f \in \mathcal{H}$ and, for each $n, f_{n} \in \mathcal{H}_{n}$. We say that the sequence $\left(f_{n}\right)$ converges to $f$ if the sequence $\left(\left\|f_{n}\right\|_{n}\right)$ is bounded and

$$
\langle g, f\rangle=\lim _{n \rightarrow+\infty}\left\langle R_{n} g, f_{n}\right\rangle_{n}
$$

for each $g \in D$. We call $f$ the limit of $\left(f_{n}\right)$ and we write $f=\lim _{n \rightarrow+\infty} f_{n}$. 
Clearly, we have $f=\lim _{n \rightarrow+\infty} R_{n} f$ for each $f \in D$. Moreover, it was shown in [1] that any $f \in \mathcal{H}$ is the limit of a sequence $f_{n} \in \mathcal{H}_{n}$ such that $\left\|f_{n}\right\|_{n}=\|f\|$ for each $n$.

The following result will be needed in Section 4 .

Proposition $3.2([1])$. Let $\left(e_{p}\right)_{p}$ be an orthonormal basis of $\mathcal{H}$. Then we can choose an orthonormal basis $\left(e_{p}^{n}\right)_{0 \leq p<p_{n}}$ of $\mathcal{H}_{n}$ for each $n$ (here $p_{n} \in$ $\mathbb{N} \cup(+\infty)$ ) in such a way that $\lim _{n \rightarrow+\infty} e_{p}^{n}=e_{p}$ for each $p$. Moreover, if we consider a sequence $f_{n} \in \mathcal{H}_{n}$ and an element $f \in \mathcal{H}$ and we write $f=\sum_{p} a_{p} e_{p}$ and $f=\sum_{p} a_{p}^{n} e_{p}^{n}$ with the understanding that $a_{p}^{n}=0$ for $p \geq p_{n}$, then $\left(f_{n}\right)$ converges to $f$ if and only if $\left(\left\|f_{n}\right\|_{n}\right)$ is bounded and $\lim _{n \rightarrow+\infty} a_{p}^{n}=a_{p}$ for each $p$.

Definition 3.3 ([3]). Let $B$ be a bounded operator on $\mathcal{H}$ and, for each $n$, let $B_{n}$ be a bounded operator on $\mathcal{H}_{n}$. We say that the sequence $\left(B_{n}\right)$ converges to $B$ if the sequence $\left(\left\|B_{n}\right\|_{\text {op }}\right)$ is bounded and if for each $f \in \mathcal{H}$ and each sequence $f_{n} \in \mathcal{H}_{n}$ with $\operatorname{limit} f$, we have $B f=\lim _{n \rightarrow+\infty} B_{n} f_{n}$.

Now we deduce from Definition 3.3 a new notion of contraction for Lie group representations. As in Section 2, we consider two real Lie groups $G$ and $H$, a group contraction $\left(c_{r}\right)_{r \in] 0,1]}$ of $G$ to $H$ and a neighborhood $V$ of $e_{H}$ as in Definition 2.2. Let $\rho$ be a unitary representation of $H$ on a Hilbert space $\mathcal{H}$ and, for each $n$, let $\pi_{n}$ be a unitary representation of $G$ on a Hilbert space $\mathcal{H}_{n}$.

Definition 3.4. We say that $\rho$ is a B-contraction of the sequence $\left(\pi_{n}\right)$ if there exists a dense space $D$ of $\mathcal{H}$, an inductive resolution $\left(\mathcal{H}_{n}, R_{n}: D \rightarrow\right.$ $\mathcal{H}_{n}$ ) of $\mathcal{H}$ and a sequence $\left.\left.r(n) \in\right] 0,1\right]$ with limit 0 such that the sequence $\pi_{n}\left(c_{r(n)}(h)\right)$ converges to $\rho(h)$ for each $h \in V$. If moreover, each operator $R_{n}$ can be extended to a continuous operator $\tilde{R}_{n}$ from $\mathcal{H}$ onto $\mathcal{H}_{n}$ such that $\left.\tilde{R}_{n}\right|_{\left(\operatorname{Ker} \tilde{R}_{n}\right)^{\perp}}$ is unitary, then we say that $\rho$ is a uB-contraction of $\left(\pi_{n}\right)$.

\section{Comparison Between Different Notions of Contraction}

In this section, we compare the notions of contractions of Lie group representations introduced in the previous sections. First, we compare the notion of MN-contraction to that of uB-contraction. These two notions are particularly adapted to the unitary setting.

As in the previous sections, we consider two real Lie groups $G$ and $H$. We assume that there exists a group contraction $\left(c_{r}\right)_{r \in] 0,1]}$ of $G$ to $H$. We also consider a unitary representation $\rho$ of $H$ on a Hilbert space $\mathcal{H}$ and, for each $n$, a unitary representation $\pi_{n}$ of $G$ on a Hilbert space $\mathcal{H}_{n}$.

Proposition 4.1. If $\rho$ is a $M N$-contraction of $\left(\pi_{n}\right)$ then $\rho$ is a $u B$-contraction of $\left(\pi_{n}\right)$. 
Proof. If $\rho$ is a MN-contraction of $\left(\pi_{n}\right)$ then we can define the operators $R_{n}$ as in Remark 2.6. Thus $\left(\mathcal{H}_{n}, R_{n}\right)$ is an inductive resolution of $\mathcal{H}$.

Let $D \subset \mathcal{H}, V \subset H$ and $(r(n))$ as in Definition 2.3. We fix $h \in V$ and in order to simplify the notation we put $B_{n}:=\pi_{n}\left(c_{r(n)}(h)\right)$ and $B:=\rho(h)$.

Fix $f \in \mathcal{H}$. Let $f_{n} \in \mathcal{H}_{n}$ be a sequence which converges to $f$. We have to prove that $\left(B_{n} f_{n}\right)$ converges to $B f$. First, we note that the norms $\left\|B_{n} f_{n}\right\|_{n}=\left\|f_{n}\right\|_{n}$ are bounded.

Let $\left(e_{p}\right)$ be an orthonormal basis of $\mathcal{H}$ consisting of elements of $D$. For each $n$, let $\left(e_{n}^{p}\right)$ be an orthonormal basis of $\mathcal{H}_{n}$ as in Proposition 3.2, that is, $\left(e_{n}^{p}\right)$ converges to $e_{p}$ for each $p$. We can write as in Proposition 3.2

$$
f_{n}=\sum_{0 \leq q<p_{n}} a_{q}^{n} e_{q}^{n}, \quad f=\sum_{q} a_{q} e_{q} .
$$

We also put

$$
c_{p q}^{n}:=\left\langle B_{n} e_{p}^{n}, e_{q}^{n}\right\rangle_{n}, \quad c_{p q}:=\left\langle B e_{p}, e_{q}\right\rangle .
$$

The proof is now divided into four steps.

1) Firstly, we note that $\lim _{n \rightarrow+\infty}\left\|A_{n}^{-1} e_{p}-e_{p}^{n}\right\|_{n}=0$ for each $p$. Indeed, we have

$$
\left\|A_{n}^{-1} e_{p}-e_{p}^{n}\right\|_{n}^{2}=\left\|A_{n}^{-1} e_{p}\right\|_{n}^{2}+\left\|e_{p}^{n}\right\|_{n}^{2}-2 \operatorname{Re}\left\langle A_{n}^{-1} e_{p}, e_{p}^{n}\right\rangle_{n}
$$

where $\left\|A_{n}^{-1} e_{p}\right\|_{n}^{2}=\left\|e_{p}^{n}\right\|_{n}^{2}=1$ and $\left\langle A_{n}^{-1} e_{p}, e_{p}^{n}\right\rangle_{n}=\left\langle R_{n} e_{p}, e_{p}^{n}\right\rangle_{n}$ converges to $\left\langle e_{p}, e_{p}\right\rangle=1$ as $n \rightarrow+\infty$ because $\left(e_{p}^{n}\right)$ converges to $e_{p}$.

2) Secondly, we show that $\lim _{n \rightarrow+\infty} c_{p q}^{n}=c_{p q}$ for each $p$ and $q$. This can be done as follows. We have

$$
\begin{aligned}
& \left\langle B_{n} e_{p}^{n}, e_{q}^{n}\right\rangle_{n}-\left\langle B_{n} A_{n}^{-1} e_{p}, A_{n}^{-1} e_{q}\right\rangle_{n} \\
& \quad=\left\langle B_{n}\left(e_{p}^{n}-A_{n}^{-1} e_{p}\right), A_{n}^{-1} e_{q}\right\rangle_{n}+\left\langle B_{n} A_{n}^{-1} e_{q}, e_{q}^{n}-A_{n}^{-1} e_{q}\right\rangle_{n} \\
& \quad+\left\langle B_{n}\left(e_{p}^{n}-A_{n}^{-1} e_{p}\right), e_{q}^{n}-A_{n}^{-1} e_{q}\right\rangle_{n} .
\end{aligned}
$$

Since the operators $B_{n}$ and $A_{n}$ are unitary, this implies that

$$
\begin{aligned}
& \left|\left\langle B_{n} e_{p}^{n}, e_{q}^{n}\right\rangle_{n}-\left\langle B_{n} A_{n}^{-1} e_{p}, A_{n}^{-1} e_{q}\right\rangle_{n}\right| \\
& \quad \leq\left\|e_{p}^{n}-A_{n}^{-1} e_{p}\right\|_{n}+\left\|e_{q}^{n}-A_{n}^{-1} e_{q}\right\|_{n} \\
& \quad+\left\|e_{p}^{n}-A_{n}^{-1} e_{p}\right\|_{n}\left\|e_{q}^{n}-A_{n}^{-1} e_{q}\right\|_{n}
\end{aligned}
$$

By using Point 1), we then obtain

$$
\lim _{n \rightarrow+\infty}\left(\left\langle B_{n} e_{p}^{n}, e_{q}^{n}\right\rangle_{n}-\left\langle B_{n} A_{n}^{-1} e_{p}, A_{n}^{-1} e_{q}\right\rangle_{n}\right)=0 .
$$

On the other hand, by Remark 2.5, we have that

$$
\lim _{n \rightarrow+\infty}\left\langle B_{n} A_{n}^{-1} e_{p}, A_{n}^{-1} e_{q}\right\rangle_{n}=\left\langle B e_{p}, e_{q}\right\rangle=c_{p q} .
$$

This gives the result. 
3) Now we will show that

$$
\lim _{n \rightarrow+\infty}\left\langle B_{n} f_{n}, e_{p}^{n}\right\rangle_{n}=\left\langle B f, e_{p}\right\rangle
$$

for each $p$. To this aim, we write

$$
\begin{gathered}
\left\langle B_{n} f_{n}, e_{p}^{n}\right\rangle_{n}=\left\langle f_{n}, B_{n}^{-1} e_{p}^{n}\right\rangle_{n}=\sum_{q}\left\langle f_{n}, e_{q}^{n}\right\rangle_{n}\left\langle e_{q}^{n}, B_{n}^{-1} e_{p}^{n}\right\rangle_{n} \\
=\sum_{q}\left\langle f_{n}, e_{q}^{n}\right\rangle_{n}\left\langle B_{n} e_{q}^{n}, e_{p}^{n}\right\rangle_{n}=\sum_{q} a_{q}^{n} c_{q p}^{n}
\end{gathered}
$$

and, similarly,

$$
\left\langle B f, e_{p}\right\rangle=\sum_{q} a_{q} c_{q p} .
$$

Now we fix $\varepsilon>0$. Let $M=1+\|f\|+\sup _{n}\left\|f_{n}\right\|_{n}$. Note that

$$
1=\left\|B^{-1} e_{p}\right\|^{2}=\sum_{q}\left|\left\langle B e_{q}, e_{p}\right\rangle\right|^{2}=\sum_{q}\left|c_{q p}\right|^{2} .
$$

Choose $q_{0}$ so that $\sum_{q>q_{0}}\left|c_{q p}\right|^{2}<\varepsilon^{2} / 9 M^{2}$. Thus we have

$$
1-\varepsilon^{2} / 9 M^{2}<\sum_{q \leq q_{0}}\left|c_{q p}\right|^{2}
$$

and using Point 2) we see that there exists $n_{1}$ so that

$$
1-\varepsilon^{2} / 9 M^{2}<\sum_{q \leq q_{0}}\left|c_{q p}^{n}\right|^{2}
$$

and hence $\sum_{q>q_{0}}\left|c_{q p}^{n}\right|^{2}<\varepsilon^{2} / 9 M^{2}$ for each $n \geq n_{1}$. Applying the CauchySchwarz inequality, we then obtain

$$
\begin{gathered}
\sum_{q>q_{0}}\left|a_{q}^{n} c_{q p}^{n}\right| \leq\left(\sum_{q>q_{0}}\left|a_{q}^{n}\right|^{2}\right)^{1 / 2}\left(\sum_{q>q_{0}}\left|c_{q p}^{n}\right|^{2}\right)^{1 / 2} \\
\leq\left\|f_{n}\right\|_{n} \varepsilon / 3 M \leq \varepsilon / 3
\end{gathered}
$$

for each $n \geq n_{1}$. Similarly, we have

$$
\sum_{q>q_{0}}\left|a_{q} c_{q p}\right| \leq\|f\| \varepsilon / 3 M \leq \varepsilon / 3 .
$$

Finally, writing

$$
\begin{gathered}
\sum_{q}\left|a_{q}^{n} c_{q p}^{n}-a_{q} c_{q p}\right| \leq \sum_{q \leq q_{0}}\left|a_{q}^{n} c_{q p}^{n}-a_{q} c_{q p}\right| \\
+\sum_{q>q_{0}}\left|a_{q}^{n} c_{q p}^{n}\right|+\sum_{q>q_{0}}\left|a_{q} c_{q p}\right|
\end{gathered}
$$

and using Proposition 3.2 and Point 2) we see that there exists $n_{2}$ so that

$$
\sum_{q}\left|a_{q}^{n} c_{q p}^{n}-a_{q} c_{q p}\right| \leq \varepsilon
$$


for each $n \geq n_{2}$. Hence (4.1) is proved.

4) By combining Point 1) and Point 3), we then obtain

$$
\lim _{n \rightarrow+\infty}\left\langle B_{n} f_{n}, A_{n}^{-1} e_{p}\right\rangle_{n}=\left\langle B f, e_{p}\right\rangle .
$$

This finishes the proof of Proposition 4.1.

The following proposition can be considered as a converse of Proposition 4.1 .

Proposition 4.2. Let $\rho$ be a uB-contraction of $\left(\pi_{n}\right)$. Assume that for each $f \in D$ there exists an integer $n(f)$ such that $\left\|R_{n} f\right\|_{n}=\|f\|$ for all $n \geq n(f)$. Then $\rho$ is a MN-contraction of $\left(\pi_{n}\right)$.

Proof. Define the operators $A_{n}$ by $A_{n}:=\tilde{R}_{n}^{-1}: \mathcal{H}_{n} \rightarrow\left(\operatorname{Ker} \tilde{R}_{n}\right)^{\perp} \subset \mathcal{H}$. The additional assumption guarantees that each $f \in D$ lies in $A_{n}\left(\mathcal{H}_{n}\right)$ for $n$ large enough. Moreover, for each $f \in D$, the sequence $A_{n}^{-1} f=R_{n} f$ converges to $f$ and then the sequence $B_{n} R_{n} f$ converges to $B f$ (here we use the same notation as in the proof of Proposition 4.1). In other words, we have

$$
\left\langle R_{n} g, B_{n} R_{n} f\right\rangle_{n}=\left\langle g, A_{n} B_{n} A_{n}^{-1} f\right\rangle \rightarrow\langle g, B f\rangle
$$

as $n \rightarrow+\infty$ for each $g \in D$. This shows that $\left(A_{n} B_{n} A_{n}^{-1} f\right)$ converges weakly to $B f$ in $\mathcal{H}$. Since $\left\|A_{n} B_{n} A_{n}^{-1} f\right\|=\|f\|=\|B f\|$, we find that $\left(A_{n} B_{n} A_{n}^{-1} f\right)$ converges strongly to $B f$. This gives the desired result.

We also have the following result.

Proposition 4.3. If $\rho$ is a nuMN-contraction of $\left(\pi_{n}\right)$ then $\rho$ is a B-contraction of $\left(\pi_{n}\right)$.

Proof. For each $n$, we fix a subspace $S_{n} \subset \mathcal{H}$ complement to $A_{n}\left(\mathcal{H}_{n}\right)$ and we define the operator $R_{n}$ by $R_{n}=A_{n}^{-1}$ on $A_{n}\left(\mathcal{H}_{n}\right)$ and $R_{n}=0$ on $S_{n}$. For each $f$ and $g$ in $D$, we have $f, g \in A_{n}\left(\mathcal{H}_{n}\right)$ for $n$ large enough. Then, recalling Definition 2.7, we have

$$
\left\langle R_{n} f, R_{n} g\right\rangle_{n}=\left\langle A_{n}^{-1} f, A_{n}^{-1} g\right\rangle_{n} \rightarrow\langle f, g\rangle
$$

as $n \rightarrow+\infty$. Hence $\left(\mathcal{H}_{n}, R_{n}\right)$ is an inductive resolution of $\mathcal{H}$.

The rest of the proof goes as in the proof of Proposition 4.1. The only change is that we have $\lim _{n \rightarrow+\infty}\left\|A_{n}^{-1} e_{p}\right\|_{n}=1$ instead of $\left\|A_{n}^{-1} e_{p}\right\|_{n}=1$ for each $n$.

\section{Examples, Remarks and Open Questions}

5.1. A contraction of $S U(1,1)$ to the Heisenberg group. Here we take $G=S U(1,1)$ and $H$ to be the 3-dimensional Heisenberg group. Let $\{X, Y, Z\}$ be a basis of $\mathfrak{h}$ in which the only nontrivial bracket is $[X, Y]=Z$. We consider the contraction of $\mathfrak{g}$ to $\mathfrak{h}$ defined by

$$
C_{r}(a X+b Y+c Z)=\frac{1}{2}\left(\begin{array}{cc}
-i r^{2} c & r(b-i a) \\
r(b+i a) & i r^{2} c
\end{array}\right)
$$


and the corresponding group contraction defined by

$$
c_{r}\left(\exp _{H}(a X+b Y+c Z)\right)=\exp _{G} C_{r}(a X+b Y+c Z) .
$$

The group $G$ acts on the unit disk $\mathbb{D}=(|z|<1)$ by linear fractional transformations. For each integer $n>2$, we consider the Hilbert space $\mathcal{H}_{n}$ of all holomorphic functions $f$ on $\mathbb{D}$ such that

$$
\|f\|_{n}^{2}:=\int_{\mathbb{D}}|f(z)|^{2} d \mu_{n}(z)<+\infty
$$

where $d \mu_{n}(z):=\frac{n-1}{\pi}\left(1-|z|^{2}\right)^{n-2} d x d y$. Here we denote by $d x d y$ the Lebesgue measure on $\mathbb{C} \simeq \mathbb{R}^{2}$. The family $f_{p}^{n}(z)=\left(\begin{array}{c}n+p-1 \\ p\end{array}\right)^{1 / 2} z^{p}, p \in \mathbb{N}$, is an orthonormal basis of $\mathcal{H}_{n}$. Let $\pi_{n}$ be the unitary representation of $G$ on $\mathcal{H}_{n}$ given by

$$
\pi_{n}(g) f(z)=(a-\bar{b} z)^{-n} f\left(g^{-1} \cdot z\right), \quad g=\left(\begin{array}{cc}
a & b \\
\bar{b} & \bar{a}
\end{array}\right) .
$$

The family $\left(\pi_{n}\right)$ is then called the holomorphic discrete series of $G=S U(1,1)$ [21].

On the other hand, we fix a real number $\lambda>0$ and we denote by $\mathcal{H}_{\lambda}$ the Hilbert space of all holomorphic functions $f$ on $\mathbb{C}$ such that

$$
\|f\|_{\lambda}^{2}:=\int_{\mathbb{C}}|f(z)|^{2} d \mu_{\lambda}(z)<+\infty
$$

where $d \mu_{\lambda}(z):=\frac{1}{2 \pi \lambda} \exp \left(-|z|^{2} / 2 \lambda\right) d x d y$. The family

$$
f_{p}^{\lambda}(z)=\frac{1}{\sqrt{(2 \lambda)^{p} p !}} z^{p}, \quad p \in \mathbb{N},
$$

is an orthonormal basis of $\mathcal{H}_{\lambda}$. Let $\rho_{\lambda}$ be the unitary irreducible representation of $H$ on $\mathcal{H}_{\lambda}$ defined by

$$
\begin{aligned}
& \rho_{\lambda}\left(\exp _{H}(a X+b Y+c Z)\right) f(z) \\
& \quad=\exp \left(i c \lambda+\frac{1}{4}(b+a i)(2 z+\lambda(-b+a i)) f(z+\lambda(-b+a i)) .\right.
\end{aligned}
$$

In [7], we showed that for each $\lambda>0, \rho_{\lambda}$ is a MN-contraction of $\left(\pi_{n}\right)$ with $r(n)=\sqrt{2 \lambda / n}$, the operators $A_{n}$ being defined by $A_{n} f_{p}^{n}=f_{p}^{\lambda}$. Then $\rho_{\lambda}$ is also a uB-contraction hence a B-contraction of $\left(\pi_{n}\right)$.

Note that another choice of the operators $A_{n}$ does not necessarily lead to a contraction result. For example, for each $n$, consider a unitary operator $B_{n}$ from $\mathcal{H}_{n}$ onto $\mathcal{H}$ so that $B_{n}^{-1} f_{0}=f_{n^{2}}^{n}$. Then for $h=\exp _{H}(c Z), c \neq 0$, we have

$$
\begin{array}{r}
\left\langle B_{n} \pi_{n}\left(c_{r(n)}(h)\right) B_{n}^{-1} f_{0}, f_{0}\right\rangle_{n}=\left\langle\pi_{n}\left(c_{r(n)}\right) f_{n^{2}}^{n}, f_{n^{2}}^{n}\right\rangle_{n} \\
=\exp \left(-i c n \frac{r(n)^{2}}{2}+i c n^{2} r(n)^{2}\right)
\end{array}
$$


which does not converge as $n \rightarrow+\infty$. Thus $\left(B_{n} \pi_{n}\left(c_{r(n)}(h)\right) B_{n}^{-1} f_{0}\right)$ does not converge in $\mathcal{H}$. Incidently, this also shows that the sequence $\left\|A_{n} \pi_{n}\left(c_{r(n)}(h)\right) A_{n}^{-1}-\rho(h)\right\|_{\text {op }}$ does not converge to 0 as $n \rightarrow+\infty$. Indeed, for $u_{n}=A_{n} B_{n}^{-1} f_{0} \in \mathcal{H}$ we have

$$
\begin{aligned}
\| A_{n} \pi_{n}\left(c_{r(n)}(h)\right) & A_{n}^{-1} u_{n}-\rho(h) u_{n} \|^{2} \\
& =2-2 \operatorname{Re}\left\langle\pi_{n}\left(c_{r(n)}(h)\right) B_{n}^{-1} f_{0}, A_{n}^{-1} \rho(h) A_{n} B_{n}^{-1} f_{0}\right\rangle \\
& =2-2 e^{i c \lambda} \operatorname{Re}\left\langle\pi_{n}\left(c_{r(n)}(h)\right) B_{n}^{-1} f_{0}, B_{n}^{-1} f_{0}\right\rangle
\end{aligned}
$$

because $A_{n}^{-1} \rho(h) A_{n} f(z)=\rho(h) f(z)=e^{i c \lambda} f(z)$ for each $f \in \mathcal{H}$. Hence $\left\|A_{n} \pi_{n}\left(c_{r(n)}(h)\right) A_{n}^{-1} u_{n}-\rho(h) u_{n}\right\|$ does not converge to 0 .

More generally, in [10], we obtained a MN-contraction of the discrete series of a semi-simple non-compact Lie group to the direct product of a Heisenberg group by an abelian group.

Similarly, in [6], we gave a MN-contraction of the unitary irreducible representations of $S U(2)$ to the representation $\rho_{\lambda}$ (see also [23]). This result was partially generalized in [9].

5.2. Some open questions. Here we mention some problems and open questions about contractions of representations which are motivated in particular by the previous examples.

(1) Let $G$ be a semi-simple non-compact Lie group. Assume that there is a group contraction of $G$ to a Lie group $H$. What unitary representations of $H$ are MN-contractions of representations of the unitary principal series and of the discrete series (if there exists) of $G$ ?

(2) Let $G$ be a semi-simple compact Lie group. Assume that there is a group contraction of $G$ to a Lie group $H$. What unitary representations of $H$ are contractions of the unitary irreducible (finitedimensional) representations of $G$ ?

(3) The problem of the 'unitarization' of the contractions of representations. In the literature, we can find some examples of nuMNcontractions. For instance, a nuMN-contraction of the discrete series of $S U(1,1)$ to some unitary representations of $\mathbb{R}^{n+1} \rtimes S O(1,1)$ was given in [12]. Then, a natural question is whether such a nuMNcontraction is also a MN-contraction. More generally, is any nuMNcontraction of unitary representations a MN-contraction?

\section{References}

[1] L. Barker, Continuum Quantum Systems as Limits of Discrete Quantum Systems, I: State Vectors, J. Funct. Anal. 186 (2001), 153-166.

[2] L. Barker, Continuum Quantum Systems as Limits of Discrete Quantum Systems, II: State Functions, J. Phys. A: Math. Gen. 22 (2001), 4673-4682.

[3] L. Barker, Continuum Quantum Systems as Limits of Discrete Quantum Systems, III: Operators, J. Math. Phys. 42 (2001), 4653-4668. 
[4] L. Barker, Continuum Quantum Systems as Limits of Discrete Quantum Systems, IV: Affine Canonical Transforms, J. Math. Phys. 44 (2003), 1535-1553.

[5] B. Cahen, Quantification d'orbites coadjointes et théorie des contractions, J. Lie Theory 11 (2001), 257-272.

[6] B. Cahen, Contraction de SU(2) vers le groupe de Heisenberg et calcul de Berezin, Beiträge Algebra Geom. 44, 2 (2003), 581-603.

[7] B. Cahen, Contraction de $S U(1,1)$ vers le groupe de Heisenberg, Mathematical works, Part XV. Luxembourg: Université du Luxembourg, Séminaire de Mathématique. (2004), 19-43 .

[8] B. Cahen, Contractions of $S U(1, n)$ and $S U(n+1)$ via Berezin quantization, J. Anal. Math. 97 (2005), 83-102.

[9] B. Cahen, Contraction of compact semisimple Lie groups via Berezin quantization, Illinois J. Math. 53 (2009), 265-288.

[10] B. Cahen, Contraction of Discrete Series via Berezin Quantization, J. Lie Theory 19 (2009), 291-310.

[11] U. Cattaneo and W. F. Wreszinski, Contractions of Lie algebra representations, Rev. Math. Phys. 11 (1999), 1179-1207.

[12] C. Cishahayo and S. de Bièvre, On the contraction of the discrete series of $S U(1,1)$, Ann. Inst. Fourier 43 (1993), 551-567.

[13] P. Cotton and A. H. Dooley, Contraction of an Adapted Functional Calculus, J. Lie Theory 7 (1997), 147-164.

[14] A. H. Dooley, Contractions of Lie groups and applications to analysis, In Topics in Modern Harmonic Analysis, Proc. Semin., Torino and Milano 1982, Vol. I , Ist. di Alta Mat, Rome, 1983, pp. 483-515.

[15] A. H. Dooley and J. W. Rice, Contractions of rotation groups and their representations, Math. Proc. Camb. Phil. Soc. 94 (1983), 509-517.

[16] A. H. Dooley and J. W. Rice, On contractions of semisimple Lie groups, Trans. Am. Math. Soc. 289 (1985), 185-202.

[17] A. Fialowski and M. Penkava, Formal Deformations, Contractions and Moduli Spaces of Lie Algebras, Internat. J. Theoretical Physics 47 (2008), 561-582 .

[18] N. A. Gromov, From Wigner-Inönü Group Contraction to Contractions of Algebraic Structures, Proceedings of the Wigner Centennial Conference, Paper No 07, Wigner Centennial Conference, Pècs, Hungary, 8-12 July, 2002.

[19] E. Inönü, E. P. Wigner, On the contraction of groups and their representations, Proc. Nat. Acad. Sci. USA 39 (1953), 510-524.

[20] A. A. Kirillov, Lectures on the Orbit Method, Graduate Studies in Mathematics Vol. 64, American Mathematical Society, Providence, Rhode Island, 2004.

[21] A. W. Knapp, Representation theory of semisimple groups. An overview based on examples, Princeton Math. Series t. 36, 1986.

[22] J. Mickelsson and J. Niederle, Contractions of Representations of de Sitter Groups, Commun. Math. Phys. 27 (1972), 167-180. 
[23] F. Ricci, A Contraction of SU(2) to the Heisenberg Group, Monatsh. Math. 101 (1986), 211-225.

[24] V. S. Varadarajan, Lie groups, Lie algebras and Their Representations, Graduate Texts in Maths 102, Springer-Verlag, 1986.

\author{
Benjamin Cahen \\ Département de Mathématiques \\ Université DE Metz, UFR-MIM \\ LMMAS, ISGMP-BÂT. A \\ Ile du Saulcy 57045, Metz Cedex 01 \\ FRANCE \\ E-mail address: cahen@univ-metz.fr
}

